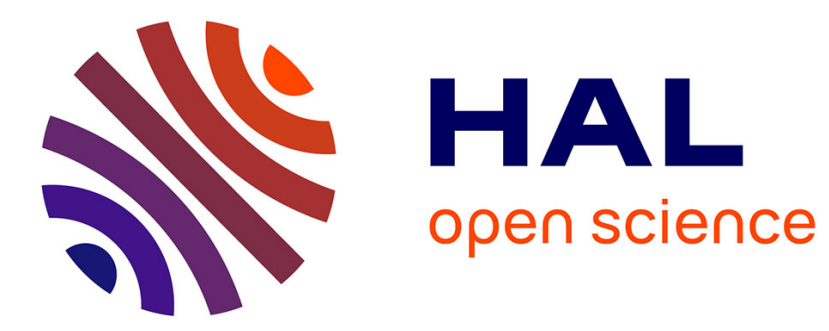

\title{
Tunability of aluminum nitride acoustic resonators: a phenomenological approach
}

Emmanuel Defay, Nizar Ben Hassine, Patrick Emery, Guy Parat, Julie Abergel, Arnaud Devos

\section{> To cite this version:}

Emmanuel Defay, Nizar Ben Hassine, Patrick Emery, Guy Parat, Julie Abergel, et al.. Tunability of aluminum nitride acoustic resonators: a phenomenological approach. IEEE Transactions on Ultrasonics, Ferroelectrics and Frequency Control, 2011, 58, pp.2516-2520. 10.1109/TUFFC.2011.2114 . hal-00783414

\section{HAL Id: hal-00783414 \\ https://hal.science/hal-00783414}

Submitted on 10 Feb 2022

HAL is a multi-disciplinary open access archive for the deposit and dissemination of scientific research documents, whether they are published or not. The documents may come from teaching and research institutions in France or abroad, or from public or private research centers.
L'archive ouverte pluridisciplinaire HAL, est destinée au dépôt et à la diffusion de documents scientifiques de niveau recherche, publiés ou non, émanant des établissements d'enseignement et de recherche français ou étrangers, des laboratoires publics ou privés.

\section{(ㄷ)(1) $\$$}

Distributed under a Creative Commons Attribution - NonCommercial| 4.0 International 


\title{
Tunability of Aluminum Nitride Acoustic Resonators: A Phenomenological Approach
}

\author{
Emmanuel Defaÿ, Nizar Ben Hassine, Patrick Emery, Guy Parat, Julie Abergel, and Arnaud Devos
}

\begin{abstract}
A phenomenological approach is developed to identify the physical parameters causing the dc-voltage-induced tunability of aluminum nitride (AIN) acoustic resonators, widely used for RF filters. The typical resonance frequency of these resonators varies from $2.038 \mathrm{GHz}$ at $-200 \mathrm{~V}$ to $2.062 \mathrm{GHz}$ at $+200 \mathrm{~V}$. This indicates, based on these RF measurements versus dc bias and the model used, that the AlN stiffness variation versus dc bias is the prominent effect because both resonance and antiresonance experience a similar variation, respectively, $24 \mathrm{MHz}$ and $19 \mathrm{MHz}$ at $400 \mathrm{~V}$. Picosecond ultrasonics were also used to prove independently that the acoustic velocity (and therefore AlN stiffness) is sensitive to dc bias and that the variation induced is comparable to that extracted from the resonance measurements. It turned out that the stiffness relative variation for an electric field of $1 \mathrm{~V} / \mu \mathrm{m}$ extracted from picosecond ultrasonics is $54 \mathrm{ppm} \cdot \mu \mathrm{m} / \mathrm{V}$. This is in good agreement with the value extracted from the RF measurements, namely $57.2 \mathrm{ppm} \cdot \mu \mathrm{m} / \mathrm{V}$. The overall tunability of these AlN resonators reaches $1.1 \%$, which is an interesting figure, although probably not high enough for genuine applications.
\end{abstract}

A LUMINUm nitride (AlN) thin films are widely used for their piezoelectric properties, especially for acoustic resonators in the gigahertz range. Their main application is RF filters for mobile phones [1]. The success of AlN is related to two main features. First, the electromechanical coupling of AlN thickness mode, $k_{t}{ }^{2}$, is around $7 \%$, which fits very well with the standard telecom bandwidth (BW) requirements for wideband code division multiple access (W-CDMA). W-CDMA typically exhibits $80-\mathrm{MHz}$ $\mathrm{BW}$ and a central frequency, $f_{0}$, of $2.14 \mathrm{GHz}\left(\mathrm{BW} / f_{0}=\right.$ $3.74 \%)$. Second, AlN exhibits a very low internal viscosity, which induces very high resonance quality factors $(Q)$. The best reported results reach 2500 around $2 \mathrm{GHz}$ [2]. It is also highly desired for the next generation of filters to exhibit tunable properties. Indeed, adding tunability would allow a dramatic simplification of the RF architectures in use. For instance, a narrow BW tunable filter (a so-called channel filter) could replace the large BW head filter, voltage-controlled oscillator ( $\mathrm{VCO}$ ), and mixer. Therefore, investigating the bias-induced frequency variation of acoustic resonators is a very interesting topic for the next generation of RF filters. Highly tunable resonators in the gigahertz range have already been reported

E. Defaÿ, N. Ben Hassine, G. Parat, and J. Abergel are with CEA LETI Minatec Campus, Grenoble, France (e-mail: edefay@cea.fr).

P. Emery and A. Devos are with the Institut d' Electronique, de Microelectronique et de Nanotechnologie, UMR CNRS 8250, Villeneuve d'Ascq, France. in the literature [3]-[5]. They all belong to the perovskite material family. Indeed, perovskite materials exhibit frequency agility that can reach $5 \%$ under $200 \mathrm{kV} / \mathrm{cm}$ dc electric field for $\mathrm{Pb}(\mathrm{Zr}, \mathrm{Ti}) \mathrm{O}_{3}(\mathrm{PZT})$ thin films [6]. In [6], the authors also reported a $k_{\mathrm{t}}^{2}$ as high as $9.5 \%$. The main drawback of all perovskite-based resonators is their low $Q$ factor compared with AlN. Indeed, for PZT, typical values are around 50 in the gigahertz range, whereas $Q$ can reach 250 for $(\mathrm{Ba}, \mathrm{Sr}) \mathrm{TiO}_{3}$ (BST) films [4]. Paraelectric BST exhibits higher $Q$ than ferroelectric PZT because there are no domain walls in the paraelectric phase. Indeed, several studies showed that the domain walls involved in purely ferroelectric materials as PZT can induce high acoustic losses and therefore low $Q$ in resonators [7] and lossy coplanar waveguide (CPW) RF lines [8]. If one considers a trade-off among all of the requirements, the best published results for tunable perovskite based resonators in the gighertz range are $Q=120, k_{\mathrm{t}}^{2}=6 \%$, and $\Delta f_{\mathrm{r}} / f_{\mathrm{r}}=$ $-2 \%$ at $E_{\mathrm{dc}}=600 \mathrm{kV} / \mathrm{cm}$ obtained with BST [5]. The perovskites tunability $s$ is mainly due to the high sensitivity of their dielectric susceptibility versus the electric field. This induces large variations of the electromechanical coupling versus the dc field. However, note that the antiresonance frequency, $f_{\mathrm{a}}$, of all acoustic resonators (and more generally of all electromechanical resonators) is independent of the electromechanical coupling as described in the IEEE Standard on Piezoelectricty [9, Eq. (60)] and explained in more detail in [10] for the special case of tunable acoustic resonators. Actually, for a piezoelectric layer alone, $f_{\mathrm{a}}$ only depends on its thickness, density, and stiffness at constant electric displacement. Therefore, the antiresonance variations are due to the stiffness sensitivity versus the applied dc field, or to couplings other than the piezoelectric coupling. Indeed, in the case of BST, Vendik et al. proposed the non-linear electrostrictive effect to explain the antiresonance frequency variations of BST resonators versus the dc field [11]. Concerning AlN tunability, it has been shown by Cheng et al. that the positions of the resonances of an AlN resonator are shifted by applying a dc voltage together with the RF signal [12]. For the antiresonance frequency, the origin of this phenomenon was attributed by Lanz et al. to a change in the elastic constant of the piezoelectric material when a bias is applied [13]. Although several authors used a thermodynamic model to explain the resonance frequency variations of perovskite materials (paraelectric or ferroelectric), this method has never been used to explain the tunability of purely piezoelectric material as AlN. In this paper, we propose to use this phenomenological approach for AlN resonators. We will show that AlN tunability is complex, 
because it exhibits many different contributions. Finally, we will prove, by using an independent experiment on the same sample (namely, picosecond ultrasonics), that the stiffness variation is indeed the main contribution to the tunability of AlN resonators when a dc bias is applied.

First, the proper variables must be chosen to describe the model. In the case of AlN piezoelectric thin-film resonators, the electric displacement $D$ is a good choice for the electrical variable because the antiresonance frequency of the thickness mode is independent of the coupling. This dramatically simplifies the model [10]. Note that this antiresonance behavior is unique and does not apply to the resonance frequency. The strain, $S$, is chosen as the mechanical variable because thin films are clamped to the substrate. Therefore, in-plane strains are zero, and because we neglect the shear strain, the out-of-plane strain is the only strain remaining, which greatly simplifies the model. Therefore, Helmholtz free energy $F$, whose natural variables are $D$ and $S$, appears to be the best choice for such a case. The free energy expansion in the case of perovskites is simplified because only the even terms are considered, for both the symmetrical and the non-symmetrical ferroelectric phases. Indeed, it is supposed that the free energy expression is very similar regardless of the phase of the perovskite considered (paraelectric or ferroelectric). Therefore, because the symmetrical paraelectric phase exhibits the simplest free energy, this is chosen in the non-symmetrical case. However, there is no reason $a$ priori to cancel out the odd coefficients of AlN free energy. $\mathrm{AlN}$ is pyroelectric and, therefore, non-symmetrical. Moreover, there is no such thing as a symmetrical prototype phase for the wurtzite phase. In this case, the expansion considered reaches the third degree as written in (1). The only dimension considered is in the thickness direction. Because the only non-zero terms of $S$ and $D$ are along the thickness direction, the free-energy expansion reduces to

$$
\begin{aligned}
F-F_{0}= & \frac{1}{2} c_{0}^{\mathrm{D}} S^{2}+\frac{1}{2} \beta_{0}^{\mathrm{S}} D^{2}-h_{0} D S+G D^{2} S+N S^{2} D \\
& +\frac{1}{3} O D^{3}+\frac{1}{3} R S^{3},
\end{aligned}
$$

where $c_{0}{ }^{\mathrm{D}}, \beta_{0} \mathrm{~S}$, and $h_{0}$ are, respectively, the stiffness coefficient at fixed $D$, impermittivity at fixed $S$, and the piezoelectric coefficient. $G$ and $N$ are both electrostrictive coefficients. $O$ and $R$ are, respectively, representative of non-linear variations of the impermittivity and of the stiffness. The partial derivatives of $F$ with respect to $S$ and $D$ are, respectively:

$$
\begin{aligned}
& \left.\frac{\partial F}{\partial S}\right)_{\mathrm{D}}=T=c_{0}^{\mathrm{D}} S-h_{0} D+G D^{2}+2 N S D+R S^{2} \\
& \left.\frac{\partial F}{\partial D}\right)_{\mathrm{S}}=E=\beta_{0}^{\mathrm{S}} D-h_{0} S+2 G D S+N S^{2}+O D^{2}
\end{aligned}
$$

Let us add a dc field to AlN by considering that this dc field is much larger than the ac field. If one only keeps the linear terms, (2) then is

$$
\begin{aligned}
& T=\left(c_{0}^{\mathrm{D}}+2 N D_{\mathrm{dc}}+2 R S_{\mathrm{dc}}\right) S+\left(-h_{0}+2 G D_{\mathrm{dc}}+2 N S_{\mathrm{dc}}\right) D \\
& E=\left(-h_{0}+2 G D_{\mathrm{dc}}+2 N S_{\mathrm{dc}}\right) S+\left(\beta_{0}^{\mathrm{S}}+2 G S_{\mathrm{dc}}+2 O D_{\mathrm{dc}}\right) D .
\end{aligned}
$$

Because the dc stress in the thickness direction is zero, the dc strain, $S_{\mathrm{dc}}$, is

$$
S_{\mathrm{dc}}=\frac{h_{0} D_{\mathrm{dc}}}{c_{0}^{\mathrm{D}}} .
$$

By substituting (4) into (3), one finally obtains:

$$
\begin{aligned}
T= & \left(c_{0}^{\mathrm{D}}+2\left(N+\frac{R h_{0}}{c_{0}^{\mathrm{D}}}\right) D_{\mathrm{dc}}\right) S \\
& +\left(-h_{0}+2\left(G+\frac{N h_{0}}{c_{0}^{\mathrm{D}}}\right) D_{\mathrm{dc}}\right) D \\
E= & \left(-h_{0}+2\left(G+\frac{N h_{0}}{c_{0}^{\mathrm{D}}}\right) D_{\mathrm{dc}}\right) S \\
& +\left(\beta_{0}^{\mathrm{S}}+2\left(\frac{G h_{0}}{c_{0}^{\mathrm{D}}}+O\right) D_{\mathrm{dc}}\right) D .
\end{aligned}
$$

These equations are actually equivalent to the $(S, D)$ piezoelectric formalism [9] by using the following substitutions (here, we considered that $D_{\mathrm{dc}}=E_{\mathrm{dc}} / \beta_{0} \mathrm{~S}$, which is true for the first order):

$$
\begin{aligned}
& h=h_{0}-2\left(G+\frac{N h_{0}}{c_{0}^{\mathrm{D}}}\right) \frac{E_{\mathrm{dc}}}{\beta_{0}^{\mathrm{S}}} \\
& c^{\mathrm{D}}=c_{0}^{\mathrm{D}}+2\left(N+\frac{R h_{0}}{c_{0}^{\mathrm{D}}}\right) \frac{E_{\mathrm{dc}}}{\beta_{0}^{\mathrm{S}}} \\
& \beta^{\mathrm{S}}=\beta_{0}^{\mathrm{S}}+2\left(\frac{G h_{0}}{c_{0}^{\mathrm{D}}}+O\right) \frac{E_{\mathrm{dc}}}{\beta_{0}^{\mathrm{S}}} .
\end{aligned}
$$

This formalism is now used to fit the frequency variations observed on the AlN resonators. The standard Mason model is used here. Each layer of the stack, simply elastic or piezoelectric, is represented by its acoustic behavior. In this paper, the AlN-based resonator is made of a Bragg mirror ( $\mathrm{SiN} / \mathrm{SiOC} / \mathrm{SiN} / \mathrm{SiOC}$ ) deposited on a 200-mm-thick Si substrate. Molybdenum bottom and top electrodes are deposited by sputtering, and $1.25-\mu \mathrm{m}-$ thick AlN is deposited by dc-pulsed sputtering. Fabrication details are given elsewhere [14]. Electrical tests are performed by adding a dc voltage to the RF signal. Because the breakdown electric field of AlN is around $5 \mathrm{MV} /$ $\mathrm{cm}$, the resonators used in this study withstand several hundred volts. The dc bias applied between top and bottom electrodes is varied between -200 and $+200 \mathrm{~V}$. A network analyzer is used to measure the $S$ parameters of the AlN resonators. The resonance, $f_{\mathrm{s}}$, and antiresonance, $f_{\mathrm{p}}$, frequencies are extracted from these data by measuring the maximum of the real parts of admittance $Y$ and impedance $Z$, respectively. Fig. 1 shows (a) $\operatorname{Re}(Y)$ and (b) $\operatorname{Re}(Z)$ versus frequency in the vicinity of the thickness 
TABle I. Extracted Parameters of the Model Obtained by Fitting the Experimental Data Shown in Fig. 1.

\begin{tabular}{lclc}
\hline$V$ bias $(\mathrm{V})$ & -200 & 0 & 200 \\
$c^{\mathrm{D}}\left(\mathrm{N} / \mathrm{m}^{2}\right)$ & $3.83 \cdot 10^{11}$ & $3.87 \cdot 10^{11}$ & $3.91 \cdot 10^{11}$ \\
$k_{\mathrm{t}}{ }^{\mathrm{S}}$ & 0.059 & 0.0577 & 0.0563 \\
$\varepsilon^{\mathrm{S}}$ & 10 & 9.7 & 9.3 \\
\hline
\end{tabular}

mode resonance versus the dc bias. Each curve is represented together with its fitting curve, as obtained from the Mason model.

One can note that $f_{\mathrm{p}}$ and $f_{\mathrm{s}}$-identified as the maximum of each curve experience an increase with the dc bias; respectively, $+0.90 \%$ and $+1.07 \%$ between -200 and $+200 \mathrm{~V}$. Moreover, the order of magnitude of the shift is similar for both $f_{\mathrm{S}}$ and $f_{\mathrm{p}}$. This is very different from what can be observed in perovskite materials, such as BST, for which $f_{\mathrm{S}}$ exhibits a shift around 5 times greater than does $f_{\mathrm{p}}$. The frequency difference between $f_{\mathrm{s}}$ and $f_{\mathrm{p}}$ only depends on the coupling factor $k_{\mathrm{t}}{ }^{2}$. Therefore, the coupling variation is much lower in AlN than in perovskites. This is confirmed by the extracted data shown in Table I. Indeed, $k_{\mathrm{t}}{ }^{2}$ experiences a shift of less than $5 \%$ versus $400 \mathrm{~V}$ bias, whereas it can change by more than one order of magnitude in BST. Physically, the main difference with perovskites is the weak dielectric constant $\varepsilon$ variation. Considering the maximum relative variation of $\varepsilon$, it reaches $7 \%$ for AlN compared with $60 \%$ for BST and $90 \%$ for PZT. This is the key to explain the low $k_{\mathrm{t}}^{2}$ variation. Because $f_{\mathrm{p}}$ does not depend on $k_{\mathrm{t}}^{2}$, its variation is only explained by stiffness variation. Because the variation is similar for $f_{\mathrm{p}}$ and $f_{\mathrm{s}}$, stiffness variation is the main parameter explaining the frequencies variations. An important point is that the quality factor of $f_{\mathrm{p}}$ and $f_{\mathrm{s}}\left(Q_{\mathrm{p}}\right.$ and $\left.Q_{\mathrm{s}}\right)$ is not affected by the bias voltage. This is an important point for tunable applications, for which it is important to have a high $Q$-factor.

Values extracted from the fitting process are $G+$ $\left(N h_{0}\right) /\left(c_{0} \mathrm{D}\right)=6.2 \cdot 10^{9}, N+\left(R h_{0}\right) /\left(c_{0} \mathrm{D}\right)=1.38 \cdot 10^{11}$, and $\left(G h_{0}\right) /\left(c_{0} \mathrm{D}\right)+O=1.14 \cdot 10^{10}$. It is very important to note that every parameter involved in these expressions matters and none of them is negligible. That means that the whole set of third-order terms involved in the free energy described by (1) must be taken into account to perform a successful fit. This is a huge difference compared with perovskites, for which the large dielectric constant variation allows many parameters to be neglected [5].

This model approach gives accurate results. It is also possible to confirm the variation of AlN stiffness by performing an independent experiment, using picosecond ultrasonics. The set-up is based on a tunable Ti:sapphire laser. Experimental details can be found elsewhere [15]. The absorption of a first optical pulse (the pump pulse) generates a thermal strain pulse whose extension can be less than $10 \mathrm{~nm}$. The resulting wave packet of longitudinal phonons propagates in the sample normally to the surface at the corresponding sound velocity. The reflection at the film/substrate interface yields returning echoes which are detected at the surface by another delayed optical pulse (the probe pulse). By adjusting the delay between pump and probe pulses, it is possible to get a very precise measurement of the round trip time, from which one can deduce the sound velocity or the film thickness [16].

For these experiments, we prepared the following stack, from the top layer to the substrate: indium tin oxide (ITO; $200 \mathrm{~nm}) / \operatorname{AlN}(1.25 \mu \mathrm{m}) / \mathrm{Mo}(150 \mathrm{~nm}) / \mathrm{Si}$ substrate. All of the layers were sputtered on a $200-\mathrm{mm}$ (100) Si substrate. The ITO layer was patterned by wet etching, providing the top electrode of the capacitors. Because the AlN and Mo layers are not patterned, there is no direct access to the bottom Mo layer. The dc voltage is therefore applied by connecting the probes to two ITO top electrodes exhibiting very different surfaces $\left(S_{\text {large }}=30\right.$ $\left.\times S_{\text {small }}\right)$. These electrodes define two in-series capacitors. Because the surface ratio is large, the voltage is mainly applied to the smallest capacitor, which is actually the device under test. The actual dc voltage range applied to the device under test is $\pm 150 \mathrm{~V}$. The area of the smallest capacitor where the laser probe is focused is $0.25 \mathrm{~mm}^{2}$. Thus, we measure the round trip time of the optically generated acoustic wave by observing the reflectivity step as seen by the probe pulse. This provides the opportunity to observe how the dc voltage $U$ affects the round-trip time. This reflectivity step corresponds to two different contri-

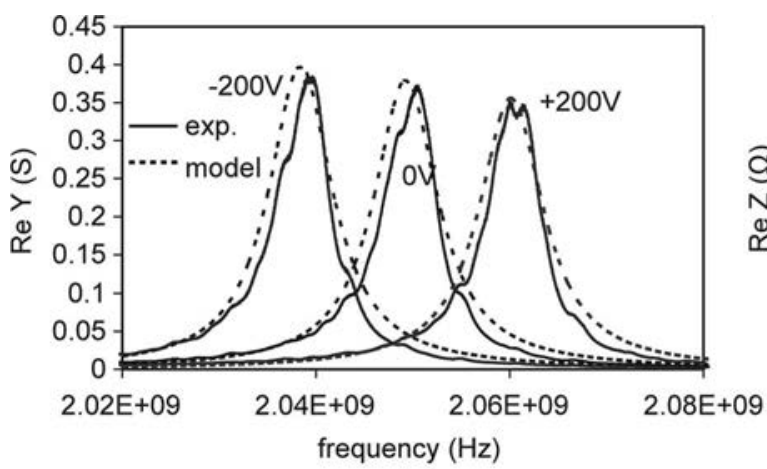

(a)

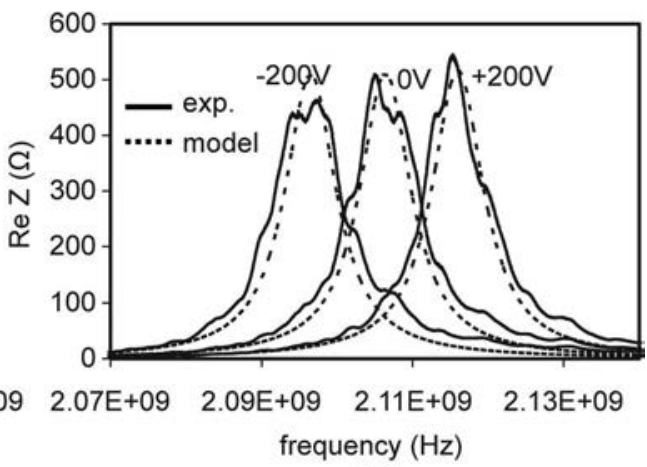

(b)

Fig. 1. (a) Real part of the admittance versus dc bias applied between -200 and $+200 \mathrm{~V}$, (b) real part of the impedance versus dc bias applied between -200 and $+200 \mathrm{~V}$. All curves are represented with their fit as obtained from the Mason model. 

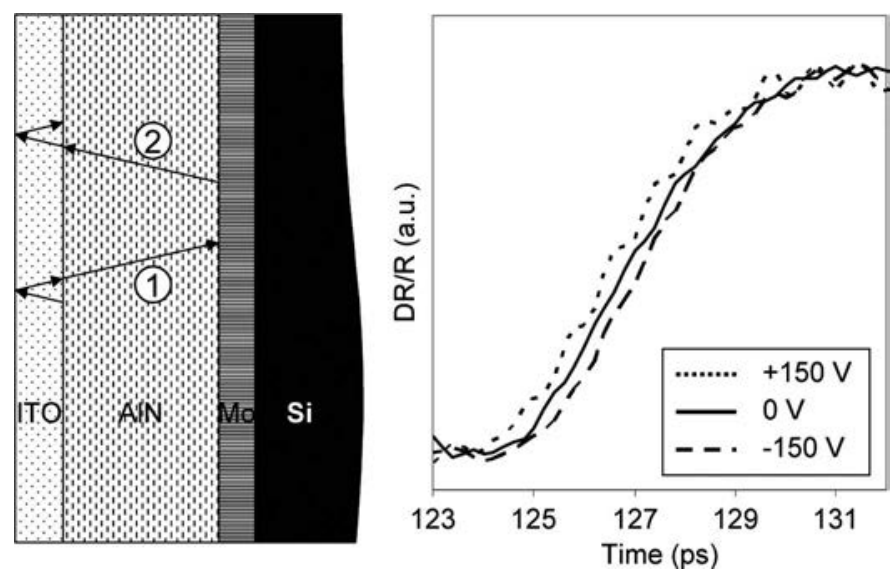

Fig. 2. (left) Cross-section of the sample and acoustic paths of the pulses that are responsible for the studied reflectivity step. (right) Magnification of the studied reflectivity step detected at three different dc voltages $(+150,0,-150 \mathrm{~V})$.

butions as shown in Fig. 2: the reverse of the pulse generated in ITO and detected at the AlN/Mo interface (path 1 in Fig. 2) and a pulse generated in the Mo and detected at the ITO/AlN interface (path 2 in Fig. 2). Both of these pulses have made one round trip in the ITO and one way through the AlN layer. We performed three experiments, at $-150,0$, and $+150 \mathrm{~V}$. Fig. 2 shows the evolution of the step's temporal position for the three different voltage conditions. One can see that the application of a positive dc voltage induces a decrease of the time of flight in AlN, assuming that ITO parameters are not affected by the electrical conditions. The relative variation of the time of flight per volt $\Delta T / T V$ is $-25 \mathrm{ppm} / \mathrm{V}$.

To go further, we have to take into account the thickness change resulting from the piezoelectric effect of AlN. The acoustic velocity of the longitudinal wave, $V_{l}$, is linked to density, $\rho$, and the stiffness coefficient $c^{\mathrm{D}}$ (that is to say, $c_{33} \mathrm{D}$ in the case of c-oriented AlN) by [10]

$$
V_{\mathrm{l}}=\sqrt{\frac{c^{\mathrm{D}}}{\rho}} .
$$

Velocity is thickness $t$ divided by the time of flight $T$, whereas density is mass $m$ divided by volume $S d$ (where $S$ is the top electrode surface area). Therefore, $c^{\mathrm{D}}$ can be written as

$$
c^{\mathrm{D}}=\frac{m t}{S T^{2}}
$$

Because the variations are very small, one can obtain the stiffness variation versus the dc bias by a basic differential calculation, which gives

$$
\frac{\mathrm{d} c^{\mathrm{D}}}{c^{\mathrm{D}}}=\frac{\mathrm{d} t}{t}-2 \frac{\mathrm{d} T}{T} .
$$

Here, we consider that the mass does not change. Moreover, the surface is also kept constant as the film is clamped on the Si substrate. The term $\mathrm{d} t / t$ is the out-ofplane strain resulting from the piezoelectric effect, which is $d_{33} E$. Because $E=-U / t,(9)$ can be written

$$
\frac{\mathrm{d} c^{\mathrm{D}}}{c^{\mathrm{D}}}=-d_{33} \frac{U}{t}-2 \frac{\mathrm{d} T}{T} .
$$

It is important to know the sign of $d_{33}$, which depends on the direction of AlN polarization $P$. In a previous study, $P$ was observed to point in the downward direction, indicating a negative sign for $d_{33}$ [14]. This induces a thickness increase when a positive bias is applied, because the electric field is also in the downward position when $U>0$. Finally, by using (10), the relative stiffness variation per volt is calculated to be $54 \mathrm{ppm} / \mathrm{V}$ by using $d_{33}$ $=-4.7 \mathrm{ppm} / \mathrm{V}$ obtained from the resonator extraction. Note that the piezoelectric term is only $4 \mathrm{ppm} / \mathrm{V}$. Because the stiffness variation is an intrinsic behavior of the material, this value is usually reported at a constant field by multiplying the results obtained by the thickness. The stiffness relative variation for an electric field of $1 \mathrm{~V} / \mu \mathrm{m}$ is therefore $54 \mathrm{ppm} \cdot \mu \mathrm{m} / \mathrm{V}$.

This result can be compared with the stiffness variation extracted from the antiresonance frequency, $f_{\mathrm{a}}$, shift. Indeed, $f_{\mathrm{a}}$ is linked to thickness $t$, stiffness at fixed $D, c^{\mathrm{D}}$, and density, $\rho$, by

$$
f_{\mathrm{a}}=\frac{1}{2 t} \sqrt{\frac{c^{\mathrm{D}}}{\rho}}=\frac{1}{2} \sqrt{\frac{c^{\mathrm{D} S}}{m t}},
$$

neglecting the electrode and Bragg mirror influence [9], [10]. By adopting the same differential calculation (also valid without taking into account electrodes and Bragg mirror because they are supposed to be unaffected by bias voltage) one obtains

$$
\frac{\mathrm{d} c^{\mathrm{D}}}{c^{\mathrm{D}}}=-d_{33} \frac{U}{t}+2 \frac{\mathrm{d} f_{\mathrm{a}}}{f_{\mathrm{a}}} .
$$

The measured relative variation of $f_{\mathrm{a}}$ versus voltage is $21 \mathrm{ppm} / \mathrm{V}$. By using $d_{33}=-4.7 \mathrm{pm} / \mathrm{V}$ and by reporting the variation for a 1- $\mu \mathrm{m}$-thick film, (12) gives $57.2 \mathrm{ppm} \cdot \mu \mathrm{m} / \mathrm{V}$ for the relative variation of stiffness for an electric field of $1 \mathrm{~V} / \mu \mathrm{m}$. This value is in very good agreement with the value obtained by picosecond ultrasonics. Lanz et al. reported $f_{\mathrm{a}}$ variation reaching $72 \mathrm{ppm} / \mathrm{V}$ for 380 -nm-thick AlN film [13]. By using this result in (12) and supposing that $d_{33}$ is identical to ours, the relative stiffness variation is calculated to be $59.4 \mathrm{ppm} \cdot \mu \mathrm{m} / \mathrm{V}$. This result is also in very good agreement with the value obtained by picosecond ultrasonics and confirmed the hypothesis proposed by Lanz that the antiresonance frequency variation versus bias is mainly due to the stiffness variation experienced by AlN. As a final remark, note that we identified the stiffness coefficient $c$ as observed by picosecond ultrasonics with $c^{\mathrm{D}}$ as characterized from the BAW antiresonance. Although the electrical conditions exhibited by picosecond ultrasonics are not very clear yet, this identification 
is mainly justified by the similar resonance and antiresonance variations. The coupling coefficient $k_{\mathrm{t}}^{2}$ reaches $7 \%$ in state-of-the-art AlN thin films, suggesting that the electrical conditions should not dramatically change the stiffness behavior of these films. This statement should obviously be reconsidered in the case of highly coupled perosvkite materials such as PZT or BST.

We can discuss these results in terms of applications and, more specifically, RF tunable resonators and filters. Indeed, if we take the results in Fig. 1, the maximum variation of the resonance and antiresonance frequencies obtained by comparing the frequencies at -200 and $+200 \mathrm{~V}$ is typically $1 \%$. Because the variation of the electromechanical coupling is also weak, the bandwidth of an RF filter made of these resonators would be almost constant. For RF filters, the ability to change the central frequency without changing the bandwidth is an interesting feature. However, the minimum desired frequency variation is around $3.8 \%$, as discussed in the introduction. This order of magnitude is almost, but not completely, reached. Another obvious drawback for applications is the huge voltage $(400 \mathrm{~V})$ required to promote this tuning. This is not affordable in a mobile phone. However, note that to generate the tuning, only the electric field matters. Therefore, decreasing the thickness of the active layer is probably something to consider, although it will increase the resonance frequency accordingly. A trade-off is perhaps achievable. It is also interesting to compare these results with the temperature variations experienced by the resonance frequencies, that is to say, the temperature coefficients of frequency (TCf) of these resonators. We obtained $\mathrm{TCf}_{\mathrm{p}}=-23 \mathrm{ppm} /{ }^{\circ} \mathrm{C}$ and $\mathrm{TCf}_{\mathrm{s}}=-21 \mathrm{ppm} /{ }^{\circ} \mathrm{C}$ [17]. Therefore, if one considers that the whole temperature range is typically $100^{\circ} \mathrm{C}$, the frequency variations reach approximately $-0.2 \%$, compared with roughly $+1 \%$ for the maximum dc bias variation. It is worth noting that these resonators are not compensated with respect to temperature. Very efficient temperature compensation can be achieved by adding silicon dioxide in the resonator stack. Such compensated AlN resonators integrated in an oscillator exhibit a temperature drift of better than $40 \mathrm{ppm}$ throughout the entire $120^{\circ} \mathrm{C}$ temperature range [18].

As a final remark, at this stage, the most interesting outcome of this study for applications is probably the following. There is a way to change the stiffness of an acoustic resonator that induces a frequency variation without notably affecting its bandwidth or its quality factor. That is to say, being able to vary stiffness is probably a very good way to achieve efficient tunable acoustic resonators.

\section{REFERENCES}

[1] R. Aigner, J. Ella, H.-J. Timme, L. Elbrecht, W. Nessler, and S. Marksteiner, "Advancement of MEMS into RF-filter applications," in Int. Electron Devices Meeting Dig., 2002, pp. 897-900.

[2] G. Fattinger, R. Aigner, and S. Marksteiner, "Everything you always wanted to know about BAW," in Workshop Proc., Asia-Pacific Microwave Conf., Yokohama, Japan, Dec. 2006.

[3] A. Volatier, E. Defay, M. Aid, A. Nhari, P. Ancey, and B Dubus, "Switchable and tunable strontium titanate electrostrictive bulk acoustic wave resonator integrated with a Bragg mirror," Appl. Phys. Lett., vol. 92, no. 3, art. no. 032906, 2008.

[4] A. Vorobiev and S. Gevorgian, "Tunable thin film bulk acoustic wave resonators with improved Q factor," Appl. Phys. Lett., vol. 96, no. 21, art. no. 212904, 2010.

[5] A. Noeth, T. Yamada, V. O. Sherman, P. Muralt, A. K. Tagantsev, and N. Setter, "Tuning of direct current bias-induced resonances in micromachined $\mathrm{Ba}_{0.3} \mathrm{Sr}_{0.7} \mathrm{TiO}_{3}$ thin-film capacitors," J. Appl. Phys., vol. 102, no. 11, art. no. $114110,2007$.

[6] J. Conde and P. Muralt, "Characterization of sol-gel PZT in thin film bulk acoustic resonators," IEEE Trans. Ultrason. Ferroelectr. Freq. Control, vol. 55, no. 6, p. 1373, 2008.

[7] P. Muralt, J. Antifakos, M. Cantoni, R. Lanz, and F. Martin, "Is there a better material for thin film BAW applications than AlN?," in IEEE Ultrasonics Symp., 2005, p. 315.

[8] E. Defaÿ, T. Lacrevaz, T. T. Vo, V. Sbrugnera, C. Bermond, M. Aid, and B. Fléchet, "Ferroelectric properties of $\mathrm{Pb}(\mathrm{Zr}, \mathrm{Ti}) \mathrm{O}_{3}$ thin films until $40 \mathrm{GHz}, "$ Appl. Phys. Lett., vol. 94, no. 5, art. no. 052901, 2009.

[9] IEEE Standard on Piezoelectricity, ANSI/IEEE Std. 176-1987, 1987, p. 32.

[10] E. Defay, Integration of Ferroelectric and Piezoelectric Thin Films. Hoboken, NJ: Wiley, 2011, p. 321.

[11] I. B. Vendika, P. A. Turalchuk, O. G. Vendik, and J. Berge, "Modeling tunable bulk acoustic resonators based on induced piezoelectric effect in $\mathrm{BaTiO}_{3}$ and $\mathrm{Ba}_{0.25} \mathrm{Sr}_{0.75} \mathrm{TiO}_{3}$ films," J. Appl. Phys., vol. 103, no. 1, art. no. $014107,2008$.

[12] Q. Cheng, T. Zhang, and Q.-M. Wang, "Frequency-temperature compensation of piezoelectric resonators by electric DC bias field," IEEE Trans. Ultrason. Ferroelectr. Freq. Control, vol. 52, no. 10, pp. 1627-1631, 2005.

[13] R. Lanz, "Piezoelectric thin films for bulk acoustic wave resonator applications: From processing to microwave filters," Ph.D. dissertation, Department of Materials, Ecole Polytechnique Fédérale de Lausanne, Lausanne, Switzerland, 2004.

[14] N. Ben Hassine, D. Mercier, P. Renaux, C. Chappaz, S. Basrour, and E. Defay, "Linear variation of aluminum nitride capacitance versus voltage induced by a piezoelectric-electrostrictive coupling," J. Appl. Phys., vol. 104, no. 3, art. no. 034110, 2008.

[15] A. Devos and R. Côte, "Strong oscillation detected by picosecond ultrasonics in silicon: Evidence for an electronic structure effect," Phys. Rev. B, vol. 70, no. 12, art. no. 125208, 2004.

[16] H. T. Grahn, H. J. Maris, and J. Tauc, "Picosecond ultrasonics," IEEE J. Quantum Electron., vol. 25, no. 12, pp. 2562-2569, 1989.

[17] N. Ben Hassine, "Etude de la fiabilité des composants à ondes acoustiques de volume (BAW) pour des applications radio fréquence," Ph.D. dissertation, Department of Techniques of Informatics and Microelectronics for Integrated Systems Architecture, Institut National de Physique de Grenoble, Grenoble, France, 2009.

[18] D. Petit, E. Cesar, P. Bar, S. Joblot, G. Parat, O. Berchaud, D. Barbier, and J. F. Carpentier, "Temperature compensated BAW resonator and its integrated thermistor for a $2.5 \mathrm{GHz}$ electrical thermally compensated oscillator," in IEEE Ultrasonic Symp., Beijing, China, 2008, p. 895.

\section{ACKNOWLEDGMENTS}

The authors thank G. Caruyer, A. Volatier, and C. Zinck for fruitful discussions at the early stages of this study. 